\title{
Evaluación de fenoles y limonoides en hojas de Cedrela odorata (Meliaceae) de una plantación experimental establecida en Tezonapa Veracruz, México
}

\author{
Samantha del Rocío Mariscal-Lucero ${ }^{1}$, Martha Rosales-Castro ${ }^{1}$, Vicente Sánchez-Monsalvo ${ }^{2} \&$ \\ José Amador Honorato-Salazar ${ }^{3}$ \\ 1. Centro Interdisciplinario de Investigación para el Desarrollo Integral Regional (CIIDIR) Unidad Durango, Instituto \\ Politécnico Nacional, Sigma 119, Durango, México; mrosa0563@yahoo.com, sam_mar9@hotmail.com \\ 2. Instituto Nacional de Investigaciones Forestales, Agrícolas y Pecuarias (INIFAP), Campo Experimental El Palmar, \\ Tezonapa Veracruz México; vicentesanche5@hotmail.com \\ 3. Instituto Nacional de Investigaciones Forestales, Agrícolas y Pecuarias (INIFAP), Campo Experimental San Martinito, \\ Puebla, México; ahonorato650@gmail.com
}

Recibido 30-VII-2014. Corregido 12-I-2015. Aceptado 30-I-2015.

\begin{abstract}
Evaluation of phenols and limonoids in leaves of Cedrela odorata (Meliaceae) from an experimental plantation established in Tezonapa Veracruz, Mexico. Cedrela odorata (Meliaceae) is a native timber tree to Tropical America, known for its high-quality wood, unfortunately, plantations of this species are severely attacked by Hypsipyla grandella. The attraction or repellency of this pest is related to secondary metabolites such as phenols and limonoids (triterpenes); therefore, it is important to study these compounds to understand the phytochemical phenomena behind this problem. With this aim, the concentration of total phenols and limonoides was evaluated in C. odorata leaves from a plantation established in Tezonapa Veracruz, Mexico. For this, a total of 66 tree leaves samples, from seven sites, were analyzed. Phenols and limonoids concentration showed significant differences not only among different provenances, but also among individual trees of the same site (Tukey, $\mathrm{p} \leq 0.05$ ). Phenols concentration was variable and in the range from 49 to $223 \mathrm{mg}$ EAG/g e for total phenols, from 7 to $158 \mathrm{mg} \mathrm{EC} / \mathrm{g}$ e for flavonoids and from 4 to $104 \mathrm{mg}$ EC/g e for proanthocyanidins. Limonoids concentration was also variable, ranging between 227 and $748 \mathrm{mg}$ EL/g e. A major compound was found by High-Performance Liquid Chromatography with Ultraviolet Diode Array Detection (HPLC-UVDAD), which corresponded to a flavonol kaempferol glycoside derivative; additionally, a flavanol catechin was also detected at low concentrations. GC-MS allowed the identification of the sesquiterpenoids $\beta$-elemene, E-caryophyllene, aromadendrene, $\alpha$-humulene, $\gamma$-cadinene, D-germacrene, bicyclogermacrene, and the poly terpenoids $\mathrm{D}$ - $\alpha$-tocopherol and $\beta$-sitosterol. Our results suggest that the evaluation of phenols may play an important role as a selection parameter for improvement and conservation programs, if they are complemented with conventional breeding practices. Rev. Biol. Trop. 63 (2): 545-558. Epub 2015 June 01.
\end{abstract}

Key words: Cedrela odorata, leaves, phenols, limonoids, HPLC, GC-MS.

Los metabolitos secundarios que producen las plantas tienen funciones ecológicas específicas, juegan un papel esencial en la protección frente a predadores, actuando como repelentes, proporcionando a la planta sabores amargos, haciéndolas indigestas o venenosas. También intervienen en los mecanismos de defensa de las plantas frente a patógenos, actuando como pesticidas naturales (Avalos,
\& Pérez-Urria, 2009). Los fenoles y limonoides pertenecen a estos tipos de compuestos. Los fenoles constituyen un amplio grupo de compuestos químicos, con diferente actividad, muchos tienen funciones importantes como defensas frente a insectos, herbívoros y hongos. Algunos compuestos fenólicos, como los flavonoides y las proantocianidinas (Andersen, \& Markham, 2006), al ser liberados en el suelo 
limitan el crecimiento de las plantas vecinas. Los limonoides son triterpenos altamente oxidados comúnmente conocidos como tetranortriterpenoides o meliacinas, dado que la familia Meliaceae se caracteriza por la biosíntesis de este tipo de compuestos de conocida actividad frente a insectos (Champagne, Koul, Isman, Scudder, \& Towers, 1992; Taylor, 1981).

Cedrela odorata L. (Meliaceae), es una especie forestal maderable comúnmente conocida como "Cedro rojo" (Spanish Cedar en inglés), nativo de América Tropical, con una amplia distribución desde los $26^{\circ} \mathrm{N}$ en México a $28^{\circ} \mathrm{S}$ en el Norte de Argentina (De la Torre, López, Yglesias, \& Cornelius, 2008; Pereyra, Inga, Santos, \& Arisméndiz, 2014). Después de la caoba, el cedro rojo es la segunda especie de madera preciosa más importante de la industria forestal de México y una de las más valiosas en los trópicos (Larrea, De los Santos, \& Hernández, 2008; Millán-Orozco, Corredoira, \& San José, 2011). Esta especie ha sido objeto de explotación con fines comerciales lo que ha ocasionado la disminución y fragmentación de las poblaciones naturales (Patiño, 1997). Por ello forma parte del Apéndice III del CITES y de la lista roja de la Norma Oficial Mexicana NOM- 059- SEMARNAT- 2010, como especie sujeta a protección especial (SEMARNAT, 2010).

El factor limitante más importante para el establecimiento exitoso de las plantaciones de $C$. odorata es el ataque de las larvas del barrenador Hypsipyla grandella (Lepidoptera: Pyralidae) (Barboza, Hilje, Durón, Cartín, \& Calvo, 2009). A pesar de que H. grandella ha sido estudiada (Cornelius \& Watt, 2003; Hilje, \& Cornelius, 2001; Pérez-Salicrup, \& Esquivel, 2008; Soto, Hilje, Mora, \& Carballo, 2011), aún no se ha desarrollado un sistema de manejo integrado. Algunos metabolitos secundarios de aceites y extractos de $C$. odorata han sido evaluados con diferente finalidad, entre ellas, la búsqueda de nuevos limonoides antialimentarios, la implicación ecológica en la atracción de $H$. grandella, evaluación de las propiedades inhibitorias de crecimiento frente algunos insectos y la investigación de sus propiedades medicinales (Asekun, \& Ekundayo, 1999; Da Silva et al., 1999; De Paula et al., 1997; González-Coloma et al., 2012; Maia et al., 2000; Kipassa et al., 2008; Martins et al., 2003; Omar et al., 2003; Silva, Souza, Rogez, Rees, \& Larondelle, 2007; Villanueva, Tuten, Haber, \& Setzer, 2009). Sin embargo, no se han evaluado las posibles diferencias cuantitativas y cualitativas de diferentes individuos de $C$. odorata. Esto es importante ya que se ha observado variabilidad en la concentración de metabolitos secundarios en plantas de diferentes procedencias y que la producción no siempre depende de condiciones climáticas del entorno (Sidhu, Kumar, \& Behi, 2003). Además, la concentración de los metabolitos secundarios en las plantas es un factor clave de sus mecanismos de defensa y, en particular la acumulación de fenoles en alguna parte de la planta representa una barrera anti-alimentaria contra insectos (Harborne, 2001).

Por lo tanto, el objetivo de este trabajo fue evaluar la concentración de fenoles totales, flavonoides, proantocianidinas y limonoides, en extractos de hojas de $C$. odorata de una plantación experimental en la que se ensayan individuos de diferentes procedencias, así como los perfiles cromatográficos de fenoles por Cromatografía Líquida de Alta Resolución (HPLC) y de terpenos por Cromatografía de Gases acoplado a Espectrometría de Masas (CG-MS), para identificar algunos de sus compuestos comunes y mayoritarios, establecer posibles diferencias entre ellos, que sirvan en un futuro como criterio para seleccionar individuos para su conservación y mejoramiento genético.

\section{MATERIALES Y METODOS}

Sitio de estudio: El estudio se llevó a cabo en una plantación de un año de edad. Los árboles fueron propagados por injerto y plantados en el Campo Experimental "El Palmar" (18 31'2"04" N - 9647'55"99" W, altitud $180 \mathrm{msnm})$, en Tezonapa, Veracruz, México. El clima del sitio es cálido-húmedo con lluvias de junio a septiembre con una precipitación media anual de $2888 \mathrm{~mm}$, y temperaturas medias de 
16 a $36^{\circ} \mathrm{C}$ en el año. La fisiografía presenta terrenos planos y lomeríos, los suelos son del tipo acrisol, profundos y de buen drenaje y poseen una textura migajón-arcillo-arenosa y pH de 4.8 (Sánchez, Salazar, Vargas, López, \& Jasso, 2003).

Recolecta de material biológico: Para este estudio se recolectaron hojas de 66 individuos de C. odorata procedentes de siete localidades: Papantla, Veracruz; Tuxtepec, Oaxaca; Teapa, Tabasco; Los Tuxtlas, Veracruz; Cárdenas, Tabasco; Calakmul, Campeche y una de la frontera México-Guatemala. El muestreo se realizó en octubre de 2012, se recolectaron aproximadamente $300 \mathrm{~g}$ de hojas frescas de cada árbol (de la misma edad), se seleccionaron hojas de tamaño uniforme con la misma apariencia y color al tiempo de la recolecta. Las hojas se secaron a temperatura ambiente a la sombra, se molieron por separado en un molino Willey hasta un tamaño de partícula de malla 60 y se almacenaron en bolsas de plástico con cierre hermético hasta su uso (Rosales-Castro et al., 2009). En el cuadro 1 se presenta la relación de las muestras recolectadas y analizadas.

Obtención de extractos: Se obtuvieron extractos metanólicos para la evaluación de fenoles y extractos acetónicos para evaluar limonoides, ya que de acuerdo a Harborne (1973) son los solventes indicados para estos compuestos.

Para la obtención de extractos metanólicos a $20 \mathrm{~g}$ de hojas secas molidas se le adicionaron $50 \mathrm{~mL}$ de metanol acuoso al $70 \%(\mathrm{v} / \mathrm{v})$, se dejaron macerar durante $48 \mathrm{hr}$ en la oscuridad y se filtraron sobre papel filtro para recuperar el extracto, al material remanente se le agregaron $25 \mathrm{~mL}$ del solvente fresco y se dejó macerar por $24 \mathrm{hr}$ en la oscuridad, después se filtró y se desechó el residuo. Los extractos obtenidos de la primera y segunda maceración se combinaron y se concentraron en un rotavapor a $45^{\circ} \mathrm{C}$ para remover el solvente. Finalmente el extracto se secó a temperatura ambiente en una campana de extracción con aire, obteniendo el extracto metanólico crudo (EMC).
De forma independiente, para los extractos acetónicos a $20 \mathrm{~g}$ de hojas secas molidas se le adicionaron $100 \mathrm{~mL}$ de acetona al $99 \%$, se dejaron macerar durante $24 \mathrm{hr}$, después de este tiempo se filtraron sobre papel filtro para recuperar el extracto, al residuo sólido remanente se le agregaron $50 \mathrm{~mL}$ de acetona y se maceraron durante $48 \mathrm{hr}$. Los extractos obtenidos de la primera y segunda maceración se combinaron y se concentraron en rotavapor a $45^{\circ} \mathrm{C}$ para remover el solvente y obtener el extracto acetónico crudo $(\mathrm{EAC})$.

Purificación de extractos: Al EMC se le realizó una purificación primaria mediante una separación líquido-líquido con acetato de etilo. A $20 \mathrm{~mL}$ del extracto metanólico crudo concentrado (después de la evaporación del solvente), se le adicionaron $2 \times 50 \mathrm{~mL}$ de acetato de etilo en un embudo de separación, la mezcla

\section{CUADRO 1}

Relación de muestras analizadas, seleccionadas de forma aleatoria

TABLE 1

Relation of analyzed samples, randomly selected

\begin{tabular}{lcc}
\multicolumn{1}{c}{ Procedencia } & Clave & $\begin{array}{c}\text { Individuos } \\
\text { seleccionados }\end{array}$ \\
Calakmul, Campeche & C8 & $1,6,14$ \\
Calakmul, Campeche & C10 & $3,14,18$ \\
Calakmul, Campeche & C11 & $1,3,4$ \\
Cárdenas, Tabasco & C62 & $3,13,21$ \\
Cárdenas, Tabasco & C67 & $3,10,16$ \\
Cárdenas, Tabasco & C46 & $7,8,9$ \\
Cárdenas, Tabasco & C64 & $5,17,24$ \\
Fron* México-Guatemala & C1 & $3,8,19$ \\
Fron* México-Guatemala & C4 & $11,14,18$ \\
Fron* México-Guatemala & C5 & $4,15,20$ \\
Papantla, Veracruz & C66 & $1,4,12$ \\
Papantla, Veracruz & C47 & $2,6,19$ \\
Teapa, Tabasco & C70 & $1,10,17$ \\
Teapa, Tabasco & C49 & $5,7,14$ \\
Teapa, Tabasco & C58 & $7,13,18$ \\
Teapa, Tabasco & C45 & $10,14,20$ \\
Tuxtepec, Oaxaca & C60 & $2,11,21$ \\
Tuxtepec, Oaxaca & C68 & $3,9,17$ \\
Tuxtepec, Oaxaca & C54 & $15,18,20$ \\
Tuxtlas, Veracruz & C55 & $5,7,14$ \\
Tuxtlas, Veracruz & C65 & $3,13,23$ \\
Tuxtlas, Veracruz & C56 & 3,68 \\
\hline
\end{tabular}

*Fron: Frontera. 
se agitó vigorosamente y se dejó en reposo para permitir la separación de las fases inmiscibles. La fracción de compuestos soluble en el acetato de etilo (fracción orgánica EMFO) se separó de la fracción insoluble, y se llevó a sequedad en rotavapor a $45^{\circ} \mathrm{C}$.

El EAC se disolvió en $2 \mathrm{~mL}$ de acetona y se colocó en una columna cromatográfica empacada con gel de sílice suspendido en diclorometano. La muestra se eluyó con diclorometano y se obtuvo la fracción uno del extracto acetónico (EAF1).

Evaluación de compuestos fenólicos: La evaluación de fenoles totales se realizó mediante el método de Folin-Ciocalteu descrito por Waterman y Mole (1994), con algunas modificaciones descritas por Rosales-Castro et al. (2012). Se utilizó una curva estándar de ácido gálico y los resultados se expresaron como equivalentes de ácido gálico por gramo de extracto seco (EAG/g e).

El contenido de flavonoides se determinó mediante el método descrito por Heimler, Vignolini, Dini y Romani (2005). Se utilizó una curva estándar de catequina, y los resultados se expresaron como equivalentes de catequina por gramo de extracto seco (EC/g e).

La determinación de proantocianidinas (flavanoles o taninos condensados) se realizó por el método propuesto de Heimler et al. (2005). Los resultados se expresaron como equivalentes de catequina por gramo de extracto $\operatorname{seco}(\mathrm{EC} / \mathrm{g}$ e).

Las determinaciones de los compuestos fenólicos se realizaron por triplicado.

Evaluación de limonoides: La evaluación de limonoides se realizó siguiendo la metodología establecida por Dai, Yaylayan, Raghavan, y Pare (1999), con una solución de vainillina $(0.02 \mathrm{mg} / \mathrm{mL})$. Se realizó una curva de calibración con limonina y los resultados se expresaron como equivalentes de limonina por gramo de extracto seco (EL/g e). En este caso solo se analizaron 22 individuos, realizando las determinaciones por triplicado.
Evaluación cromatográfica: Para la obtención de los perfiles cromatográficos de fenoles se utilizó HPLC, en un cromatógrafo marca Perkin Elmer, serie 200 con detector UV de arreglo de diodos (UV-DAD), columna $\mathrm{C}_{18}$, $5 \mu \mathrm{m}$ de poro, $4.6 \mathrm{~mm}$ por $250 \mathrm{~mm}$. Los extractos EMFO se disolvieron en metanol grado HPLC y se inyectaron $20 \mu \mathrm{L}$ de muestra. Como sistema de elución se utilizó como fase A agua acidificada con ácido fosfórico a un $\mathrm{pH}$ de 2.9 y como fase B acetonitrilo, flujo de $1 \mathrm{~mL} / \mathrm{min}$. La elución se inició con $100 \%$ de A y finalizó con $40 \%$ de $\mathrm{B}$ en un tiempo de $40 \mathrm{~min}$. Se midieron a longitudes de onda de 280 y $360 \mathrm{~nm}$. Como estándar de referencia se utilizó solución de catequina a $1000 \mathrm{mg} / \mathrm{L}$.

Para la evaluación cromatográfica de los extractos acetónicos, los extractos EAF1 y EAC fueron analizados mediante CG-MS, en un cromatógrafo marca Hewlett-Packard (Agilent) serie HP-5890 con un detector de ionización de flama (FID). Se utilizó una columna Agilent DB-5MS de $30 \mathrm{mx} 0.25 \mathrm{~mm}, 0.25 \mu \mathrm{m}$ de diámetro interno. Se utilizó helio como gas de arrastre y una temperatura de inyección de $250^{\circ} \mathrm{C}$.

Los resultados de la evaluación de compuestos fenólicos y limonoides se analizaron con el programa STATISTICA 7 (StatSoft, 2004), se realizó un análisis de varianza con una comparación múltiple de medias por el método de Tukey $(\mathrm{p} \leq 0.05)$, un análisis de correlación de Pearson y un análisis multivariado (análisis de clúster por el método de Ward, análisis de componentes principales y conglomerados K-medias) para determinar las diferencias estadísticas entre las concentraciones de los individuos de diferentes procedencias y lograr una agrupación de acuerdo a las características particulares de los individuos estudiados.

En el perfil cromatográfico de EMFO se realizó una matriz de presencia-ausencia de compuestos en los diferentes tiempos de retención observados en los cromatogramas de cada muestra en particular. A esta matriz se le efectuó un análisis de conglomerados mediante el 
índice de Jaccard utilizando el programa PAST versión 1.34 (Hammer, Harper, \& Ryan, 2001).

\section{RESULTADOS}

Evaluación de fenoles: Las concentraciones de fenoles y flavonoides totales se muestran en figura 1. El ANOVA indicó diferencias significativas (Tukey, $\mathrm{p} \leq 0.05$ ) entre de las concentraciones de los fenoles totales de los 66 individuos analizados de las siete procedencias. Los extractos de los individuos de la procedencia de Calakmul presentaron las mayores concentraciones, con valores de hasta $223 \mathrm{mg}$
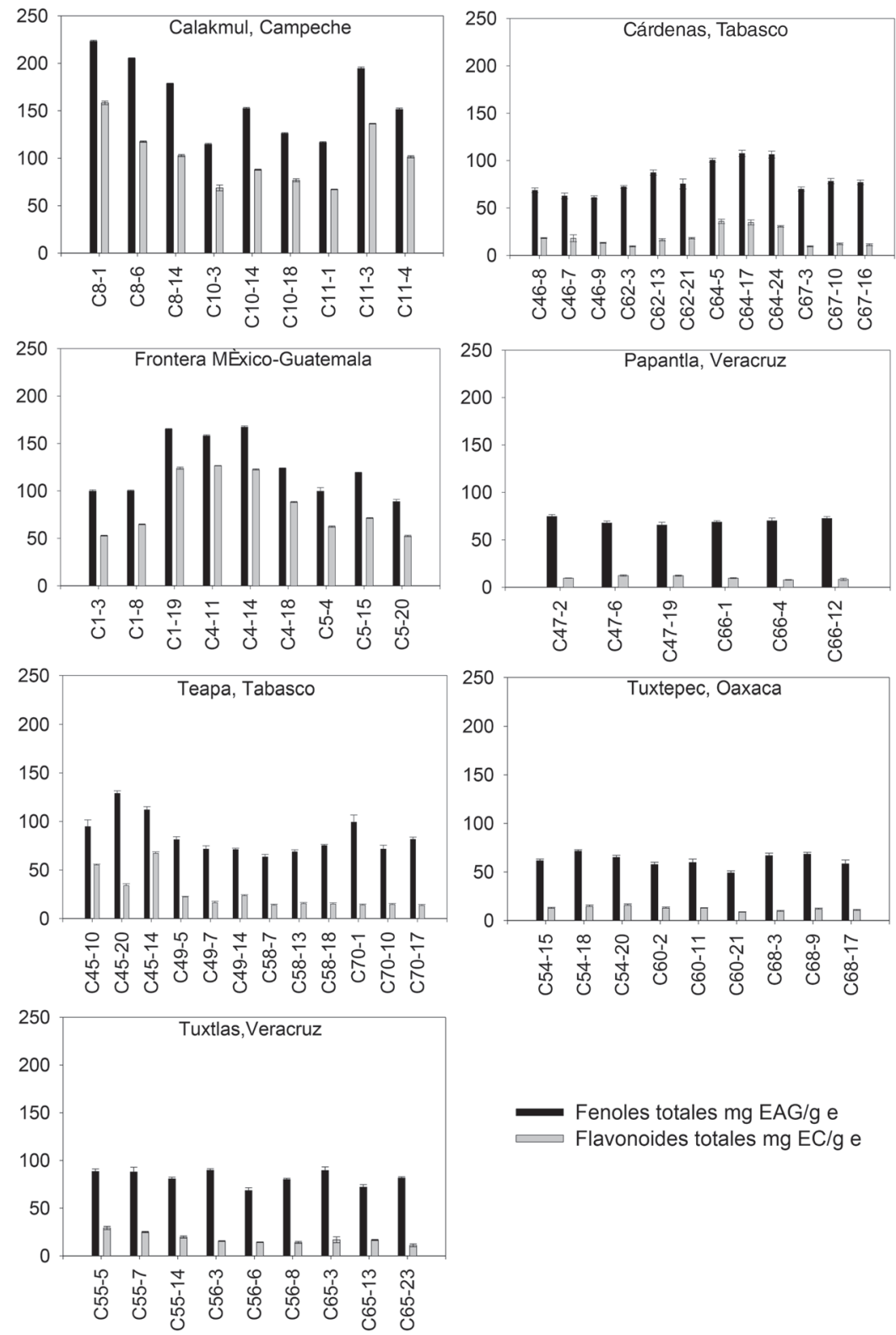

Fig. 1. Concentración de fenoles totales y flavonoides, en hojas de $C$. odorata, de siete procedencias. Fig. 1. Total phenols and flavonoids concentration in $C$. odorata leaves from seven provenances. 
EAG/g e del individuo C8-1 seguido por C8-6 con 206mg EAG/ge. Las menores concentraciones de fenoles totales se obtuvieron en los extractos de los individuos de la procedencia de Tuxtepec con un valor de 49mg EAG/g e del individuo C60-21.

El ANOVA de la concentración de flavonoides mostró diferencias estadísticas significativas (Tukey, $\mathrm{p} \leq 0.05$ ) entre individuos $\mathrm{y}$ procedencias. Las mayores concentraciones de flavonoides se observaron en los individuos de la procedencia de Calakmul y Frontera México-Guatemala con valores máximos de 158 y 126mg EC/g e de los individuos C8-1 y C4-11, respectivamente. Las menores concentraciones de flavonoides se presentaron en las procedencias de Papantla y Tuxtepec, con valores mínimos de 7mg EC/g e para el individuo C66-4 y un valor de $8 \mathrm{mg}$ EC/g e para C60-21, (Fig. 1).

Las concentraciones de proantocianidinas (Fig. 2A) presentaron diferencias significativas entre individuos y procedencias (Tukey, $\mathrm{p} \leq 0.05$ ). La mayor concentración es $104 \mathrm{mg}$ $\mathrm{EC} / \mathrm{g}$ e del individuo de Calakmul C8-1, seguido por el C1-19 de Frontera México-Guatemala con un valor de $72 \mathrm{mg} \mathrm{EC/g}$ e, las menores

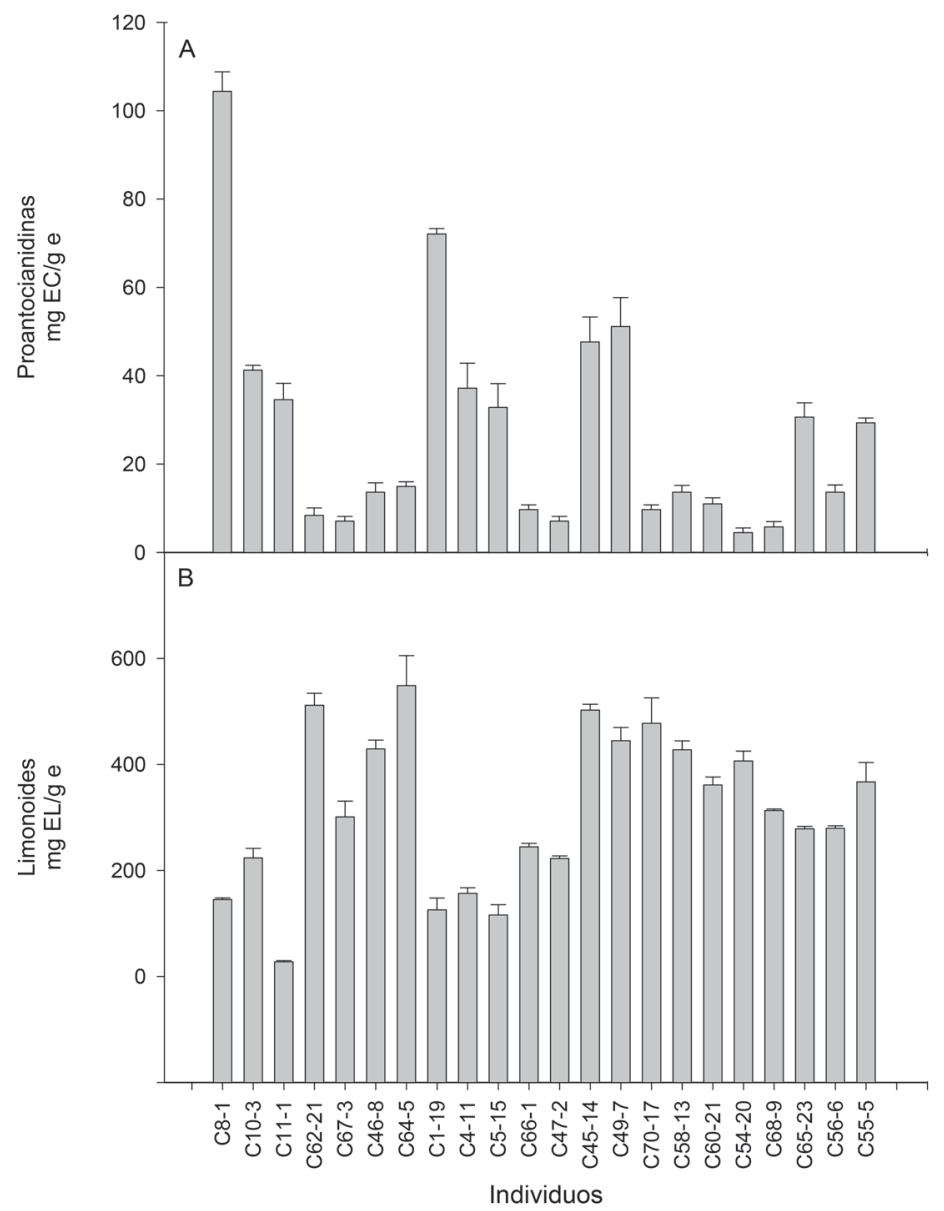

Fig. 2. (A) Concentración de proantocianidinas en hojas de C. odorata. (B) Concentración de limonoides en hojas de $C$. odorata.

Fig. 2. (A) Concentration of Proanthocyanidins in C. odorata leaves. (B) Concentration of limonoides in C. odorata leaves. 
concentraciones se presentaron con los valores de 6 y $4 \mathrm{mg}$ EC/g e de los individuos C68-9 y C54-20 de la procedencia de Tuxtepec.

El análisis de correlación (Pearson, $\mathrm{p} \leq 0.05$ ) entre la concentración de fenoles totales y flavonoides mostró una correlación significativa y positiva entre estas dos variables $\left(r^{2}=0.88\right)$. Asimismo, se observó una correlación significativa (Pearson, $\mathrm{p} \leq 0.05$ ) entre fenoles totales, flavonoides y proantocianidinas $\left(r^{2}=0.7\right)$, por lo que a mayor concentración de fenoles totales hubo una mayor concentración de flavonoides y proantocianidinas en los individuos evaluados.

Evaluación de limonoides: Los resultados de la evaluación de limonoides se muestran en la figura 2B. El ANOVA presentó diferencias estadísticamente significativas (Tukey, $\mathrm{p} \leq 0.05$ ). Las mayores concentraciones de limonoides se presentaron en los individuos de la procedencia de Cárdenas, con valores de $748 \mathrm{mg}$ EL/g e para C64-5 y $711 \mathrm{mg}$ EL/g e en C62-21, seguidos por el individuo de Tuxtepec C45-14 con una concentración de 702mg EL/g e. La concentración menor se observó en el individuo de Calakmul C11-1 con un valor de $227 \mathrm{mg}$ EL/g e. El análisis de correlación (Pearson, $p \leq 0.05$ ) entre la concentración de limonoides y fenoles totales no mostró una correlación significativa entre estas variables $\left(r^{2}=0.3\right)$.

La aplicación de las tres técnicas del análisis multivariado a las cuatro variables de estudio (fenoles totales, flavonoides, proantocianidinas y limonoides) permitió inferir el agrupamiento de los individuos analizados en tres grupos, los cuales se observan en la figura 3 .

Evaluación cromatográfica: $\mathrm{El}$ análisis de los cromatogramas de HPLC (Fig. 4A) indicó semejanza química entre los extractos

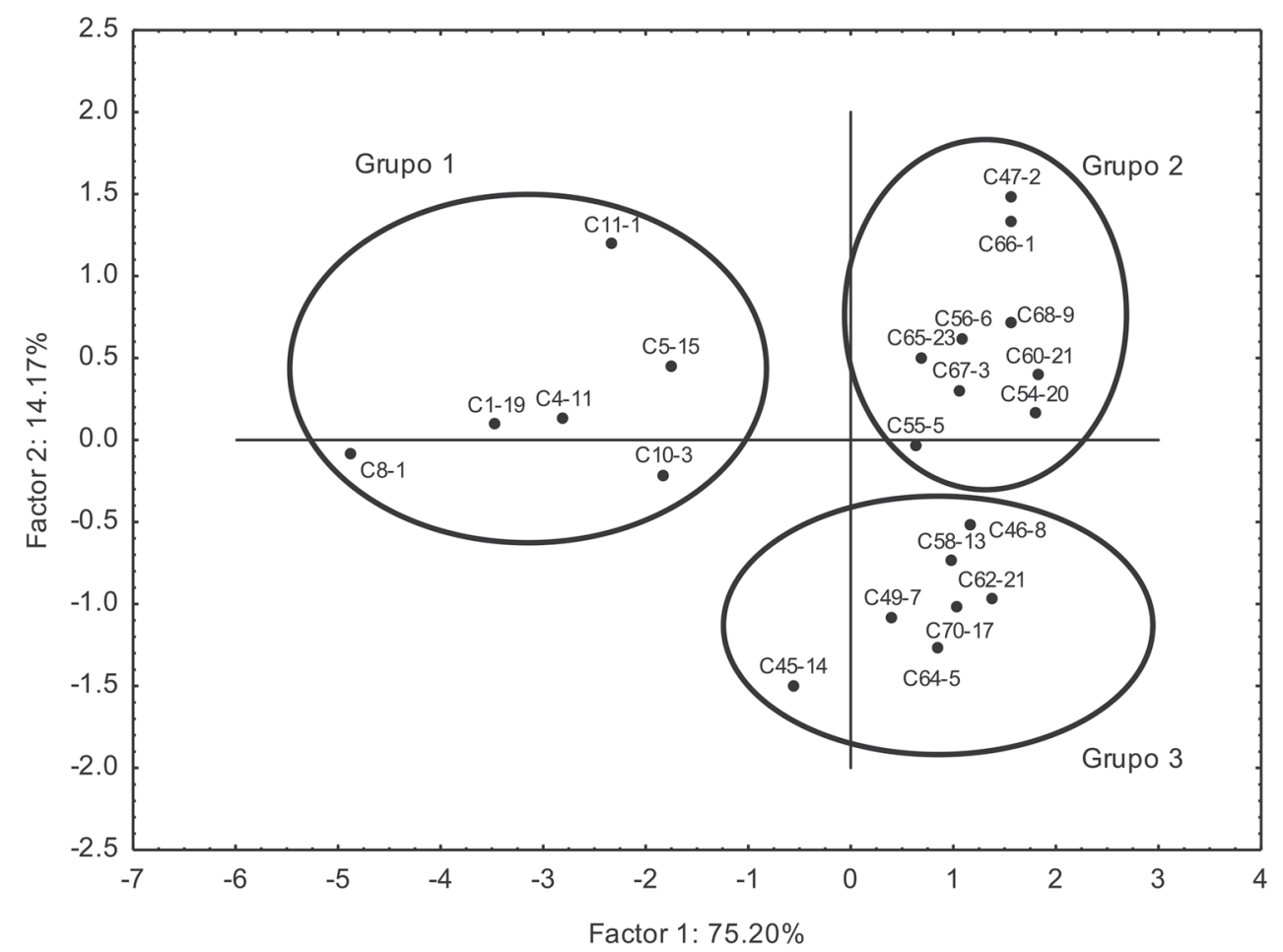

Fig. 3. Agrupamiento de los individuos de C. odorata, en función de los dos componentes principales obtenidos con la matriz de correlación de las cuatro variables (fenoles totales, flavonoides, proantocianidinas y limonoides).

Fig. 3. Grouping of $C$. odorata individuals, depending on two principal components obtained from the correlation matrix of the four variables (total phenols, flavonoids, proanthocyanidins and limonoids). 


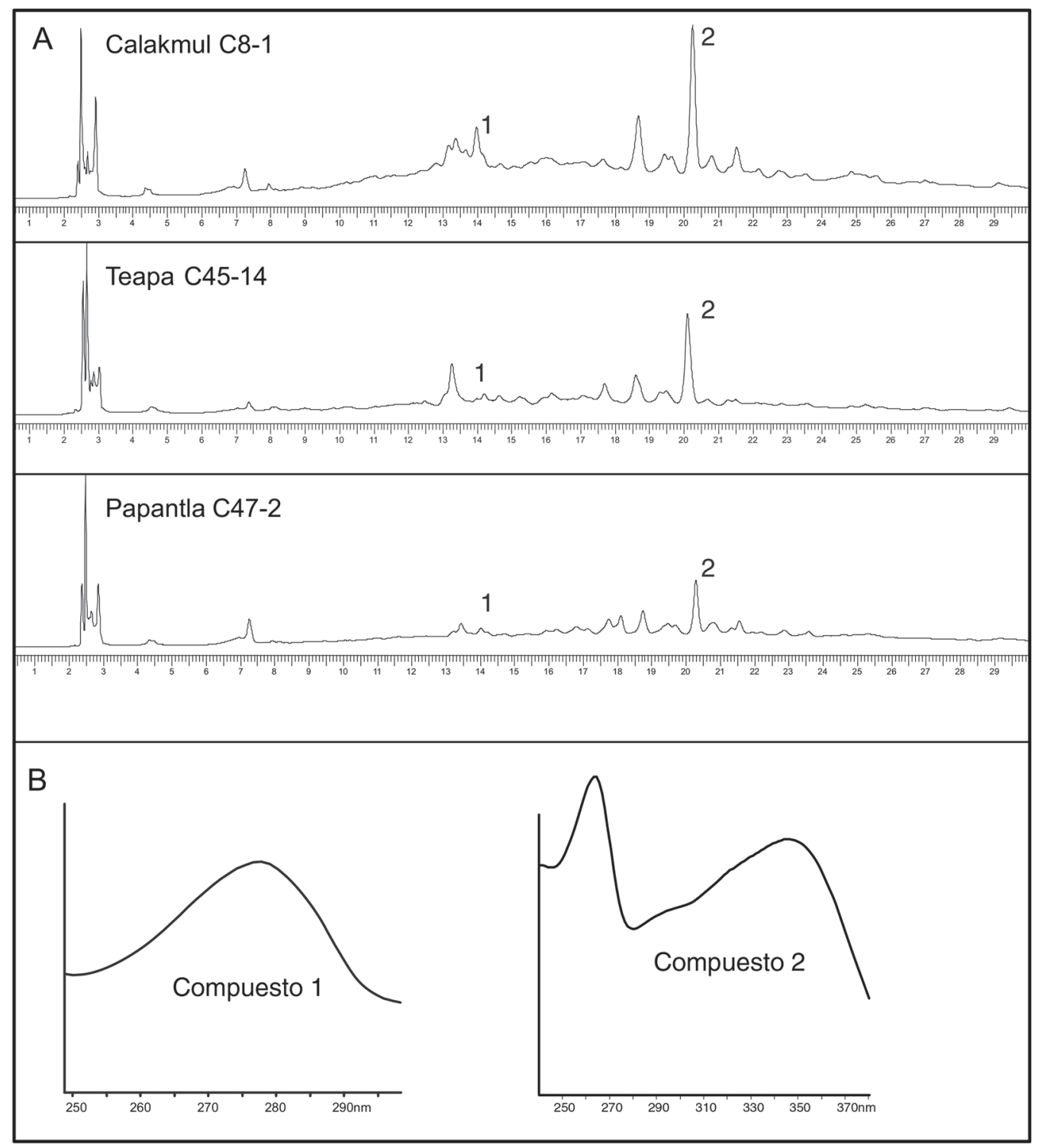

Fig. 4. (A) Cromatogramas de HPLC de extractos metanólicos fracción orgánica (EMFO) de hojas de C. odorata. (B) Espectros de UV del compuesto 1 (catequina) y 2 (glicósido de kaempferol).

Fig. 4. (A) HPLC chromatograms of methanol extracts organic fraction (EMFO) of $C$. odorata leaves. (B) UV spectra of compound 1 (catechin) and 2 (kaempferol glycoside).

fenólicos evaluados de las diferentes procedencias, destacando la presencia de un compuesto mayoritario que eluyó a un tiempo de $20.2 \mathrm{~min}$ (Fig. 4A compuesto 2), este compuesto se observó en todos los individuos sólo que a diferentes concentraciones. El espectro ultravioleta de este compuesto presentó absorbancias máximas de 265 y 350nm (Fig. 4B compuesto 2 ), señales que corresponden a un compuesto flavonoide del grupo flavonol, de tipo glicósido de kaempferol.

En los cromatogramas se identificó también la presencia de catequina, con tiempo de retención de 13.9min (Fig. 4A compuesto 
1), este compuesto se identificó con base a su espectro de UV (Fig. 4B compuesto 1), así como por la comparación del tiempo de retención con el estándar de catequina.

En el análisis de conglomerados, de la matriz binaria resultante de los perfiles cromatográficos de HPLC, se presentaron tres grupos claramente diferenciados (Fig. 5).

Los resultados de CG-MS se presentan en el cuadro 2, se identificó la presencia de los compuestos mediante la comparación de los espectros de masas obtenidos, con los espectros de la base de datos del equipo de CG-MS utilizado. Se identificaron los siguientes compuestos: $\beta$-elemeno, E-cariofileno, aromadendreno, humuleno, D-germacreno, Di- $\alpha$-Tocoferol y $\beta$-sitosterol.

\section{DISCUSIÓN}

Los árboles de C. odorata evaluados resultaron ampliamente variables en cuanto a sus concentraciones de fenoles y limonoides observándose diferencias marcadas entre individuos y procedencias. Los individuos de
CUADRO 2

Compuestos identificados por CG-MS en extractos acetónicos de hojas de $C$. odorata

TABLE 2

Identified compounds by GC-MS in acetone extracts of $C$. odorata leaves

\begin{tabular}{clc}
$\mathrm{TR}^{*}(\min )$ & \multicolumn{1}{c}{$\mathrm{EAF}{ }^{*}$} & $\mathrm{EAC}^{*}$ \\
8.0 & $\beta$-elemeno & - \\
8.7 & E-Cariofileno & - \\
9.1 & Aromadendreno & - \\
9.5 & $\alpha$-Humuleno & - \\
9.8 & Gama-cadineno & - \\
10.0 & D-Germacreno & D-Germacreno \\
10.4 & Biciclogermacreno & - \\
39.2 & Di- $\alpha$-Tocoferol & Di- $\alpha$-Tocoferol \\
41.6 & $\beta$-sitosterol & $\beta$-sitosterol \\
\hline
\end{tabular}

*TR: Tiempo de retención, EAF1: Fracción uno del extracto acetónico, EAC: Extracto acetónico crudo.

la procedencia Calakmul, Campeche presentaron los valores más altos de fenoles totales, flavonoides y proantocianidinas, pero menor concentración de limonoides. Por otro lado, las concentraciones más bajas de fenoles se obtuvieron en las procedencias de Tuxtepec, Oaxaca y Papantla, Veracruz. En cuanto a las
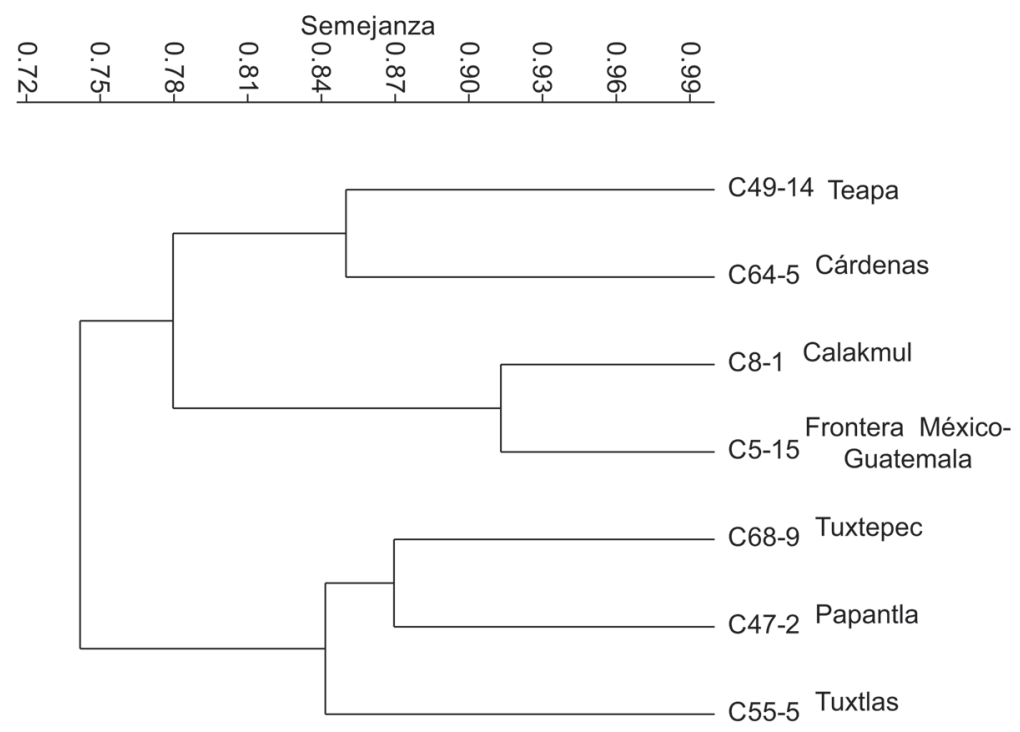

Fig. 5. Análisis de clúster de la semejanza cromatográfica de los extractos fenólicos de hojas de $C$. odorata, con base en el índice de Jaccard.

Fig. 5. Cluster analysis of the chromatographic similarity of phenolic extracts of $C$. odorata leaves, based on the Jaccard index. 
concentraciones más elevadas de limonoides se obtuvieron en las procedencias de Cárdenas y Teapa, Tabasco. Estas diferencias se observan en el análisis de agrupamiento, con la formación de tres grupos claramente diferenciados en cuanto a la concentración de los fenoles y limonoides. El primer grupo está formado por los individuos de Calakmul, Campeche y los individuos de la Frontera México-Guatemala, estos individuos tienen la característica de presentar altas concentraciones de fenoles y bajas en limonoides. El segundo grupo está formado por los individuos de Papantla, Veracruz, Tuxtepec, Oaxaca y Tuxtlas, Veracruz, los cuales presentan las concentraciones más bajas de fenoles. Finalmente, el tercer grupo está conformado por los individuos de Teapa y Cárdenas, Tabasco caracterizados por altas concentraciones de limonoides.

La correlación $\left(\mathrm{r}^{2}=0.7\right)$ que se observa entre los fenoles, flavonoides y proantocianidinas se debe a que los flavonoides son un grupo importante y numeroso dentro de los compuestos fenólicos y a la vez, las proantocianidinas pertenecen al grupo de los flavonoides (flavanoles) por lo cual están relacionados de manera proporcional. Se observa que los individuos que producen mayor concentración de fenoles producen una menor concentración de limonoides, sin embargo, dicha correlación no es estadísticamente significativa $\left(\mathrm{r}^{2}=0.30\right)$. Lo anterior sugiere que la composición de los metabolitos secundarios evaluados en $C$. odorata en el presente trabajo tienen características propias de acuerdo a la procedencia. En este sentido, se ha documentado que los fenoles están directamente involucrados en la resistencia a insectos en muchas plantas (Harbone, 2001). Ramputh, Teshome, Bergvinson, Nozzolillo y Arnason (1999) encontraron una relación directa entre la alta concentración de fenoles en el grano de diferentes genotipos de sorgo y la resistencia al ataque de Sitophilus oryzae (Coleoptera: Curculionidae), en este estudio se propuso al contenido fenólico como un indicador de resistencia. Del mismo modo, Pérez-Flores, Eigenbrode y Hilje-Quiroz (2012) obtuvieron extractos fenólicos de $C$. odorata capaces de disminuir el crecimiento de la larva de $H$. grandella. Los anterior sugiere que los individuos que forman al grupo uno pueden llegar a presentar una mejor defensa frente al ataque de $H$. grandella. Por otro lado, algunas investigaciones reportan que los limonoides son de poco valor en la resistencia al barrenador (Da Silva et al., 1999; De Paula et al., 1997), sin embargo, los limonoides de la familia Meliaceae han sido estudiados por sus propiedades insecticidas, como el estudio de Kipassa et al. (2008), quienes hacen referencia a los limonoides de la corteza del tallo de $C$. odorata y la capacidad anti-alimentaria en Spodoptera littoralis (Lepidoptera: Noctuidae). De esta forma, los individuos del grupo tres resultan interesantes como fuente de limonoides para investigaciones posteriores con respecto a sus propiedades insecticidas.

Los perfiles cromatográficos de los compuestos fenólicos de los individuos de $C$. odorata mostraron semejanza con respecto a la presencia y/o ausencia de los compuestos de acuerdo a sus cromatogramas, el análisis de clúster indica la presencia de tres grupos: un grupo integrado por los individuos de Papantla, Tuxtepec, y Tuxtlas, separado completamente de los dos siguientes grupos: un grupo formado por los individuos de Calakmul y Frontera México-Guatemala, y el otro por los individuos de Cárdenas y Teapa. Estos grupos coinciden con el análisis de agrupamiento evaluado en el punto anterior, lo que sugiere que las diferencias en concentración pudieran estar relacionadas con la presencia o ausencia de algunos compuestos fenólicos en los individuos analizados. En este aspecto, Macías-Sámano (2001) señala que mediante programas de mejoramiento genético se podrían ensayar individuos de $C$. odorata de diferentes procedencias para la búsqueda de compuestos de interés. El compuesto fenólico mayoritario y presente en todas las muestras analizadas, se infiere que corresponde a un flavonol de tipo glicósido de kaempferol, por el espectro de UV ( $\lambda$ máx 265 y $350 \mathrm{~nm}$ ), comparando con el espectro que reportan Campos \& Markham (2007). Compuestos de este tipo han sido identificado 
anteriormente en $C$. odorata (Pérez-Flores et al., 2012). De igual forma, en hojas de Cedrela sinensis (Meliaceae) se ha encontrado un glicósido de kaempferol con actividad antioxidante importante (Lee et al., 2006). Se identificó también el flavanol catequina, tanto por comparación del tiempo de retención con el estándar (13.9min), como por el espectro de UV $(\lambda$ máx 278nm), aunque en algunos individuos está presente en una concentración muy baja. La catequina es un precursor de las proantocianidinas o taninos condensados, en los individuos de procedencias Calakmul C8-1, Frontera México-Guatemala C1-19, Teapa C45-14 y C49-7 se obtuvieron altas concentraciones de proantocianidinas, por lo que puede inferirse que sea alto el contenido de catequina en estos individuos. De Paula et al. (1997) reportaron no haber encontrado catequina en $C$. odorata y que este flavanol se translocó de $T$. cilata (Meliaceae) a $C$. odorata por injerto, además señalan a este compuesto como el posible responsable de la resistencia de $T$. ciliata al barrenador $H$. grandella, sin embargo en el presente estudio se identificó el flavanol catequina en hojas de $C$. odorata, tanto de forma monomérica identificada por HPLC, como también por la evaluación cuantitativa de proantocianidinas, las cuales están formadas por unidades de catequina y otros flavan-3-oles, (Rosales-Castro et al.(2012). Mediante CG-MS se identificaron los sesquiterpenos $\beta$-elemeno, E-cariofileno, aromadendreno, humuleno, gama cadineno y D-germacreno, y los poli terpenos $\mathrm{Di}-\alpha$ Tocoferol y $\beta$-sitosterol. Los sesquiterpenos identificados en las hojas de $C$. odorata en este estudio han sido reportados en aceite esencial de hojas y tallo para esta misma especie (Maia et al., 2000; Villanueva et al., 2009). Por el contrario, no se encontraron evidencias de la presencia de triterpenoides del tipo Mexicanoide en las muestras analizadas, tales como gedunina o azadiractina, que han sido reportadas para especies de Cedrela spp. (Céspedes, Calderón, Lina, \& Aranda, 2000).

La variabilidad encontrada en los fenoles y limonoides en los individuos de la diferentes procedencias, coinciden con la variación encontrada en diversas características de $C$. odorata. En este sentido, Sánchez et al. (2003) encontraron variación en cuanto a parámetros de crecimiento en $C$. odorata de diferentes procedencias de México. Así mismo, hay evidencia en los ensayos de progenie y procedencia que indican que algunos genotipos muestran diferentes niveles de tolerancia al ataque del barrenador (Newton, Leakey, \& Mesean, 1993, Newton, Cornelius, Mesen, \& Leakey, 1995; Newton et. al., 1999). De igual forma, se ha demostrado que $C$. odorata presenta mayor variabilidad genética que otras especies del género Cedrela. Muellner, Pennington, y Chase (2009) reportaron diferencias genéticas en $C$. odorata de acuerdo a su hábitat a lo largo del neotrópico. Por otro lado, Navarro, Ward y Hernández (2002) estudiaron plántulas de $C$. odorata de diferentes hábitat distribuidas por toda Costa Rica encontrando diferencias morfológicas entre estas.

La producción de diferentes metabolitos secundarios en $C$. odorata podrían jugar un papel importante como parámetro de selección en programas de conservación y mejoramiento, al complementar las prácticas convencionales de mejoramiento genético. Por lo tanto, los individuos de $C$. odorata procedentes de Calakmul podrían ser incorporados a programas de conservación y mejoramiento genético. Por último, sugerimos la búsqueda de individuos con altas concentraciones de catequina dada a la importancia que se reporta de este flavonoide con respecto a la resistencia sobre H. grandella.

\section{AGRADECIMIENTOS}

Este proyecto fue financiado por el Fondo Sectorial CONACYT-CONAFOR, proyecto 2010-C01-134514. El primer autor agradece al Consejo Nacional de Ciencia y Tecnología (CONACYT) y al Instituto Politécnico Nacional (IPN) por el apoyo para realizar estudios de Doctorado en Ciencias en Biotecnología. Agradecemos al Instituto Nacional de Investigaciones Forestales, Agrícolas y Pecuarias (INIFAP) por las facilidades prestadas para la 
realización de este trabajo, así como también a la Universidad Estatal de Oregón (OSU)- USA por el apoyo para el análisis de CG-MS.

\section{RESUMEN}

Cedrela odorata (Meliaceae) es una especie forestal maderable nativa de América Tropical, conocida por la alta calidad de su madera. Plantaciones de esta especie son atacadas severamente por Hypsipyla grandella; la atracción o repelencia de la plaga está relacionada con metabolitos secundarios tipo fenoles y limonoides (triterpenos), por lo que el estudio de estos compuestos es importante para comprender algunos fenómenos fitoquímicos. Se evaluó la concentración de fenoles totales y limonoides en hojas de C. odorata (Meliaceae) de una plantación establecida en Tezonapa Veracruz México, se analizaron 66 individuos de siete procedencias. La concentración de fenoles y limonoides mostró diferencias significativas, no solo entre las procedencias sino también entre los árboles de una determinada procedencia (Tukey, $\mathrm{p} \leq 0.05$ ). La concentración de fenoles totales varió de 49 a $223 \mathrm{mg}$ EAG/g e, los flavonoides de 7 a $158 \mathrm{mg}$ EC/g e y las proantocianidinas de 4 a $104 \mathrm{mg} \mathrm{EC/g} \mathrm{e,} \mathrm{mientras} \mathrm{que} \mathrm{en} \mathrm{limonoides} \mathrm{se} \mathrm{obtuvieron}$ valores de 227 a $748 \mathrm{mg}$ EL/g e. Mediante Cromatografía Líquida de Alta Resolución con detector UV-Arreglo de Diodos (HPLC-UV-DAD) se encontró un compuesto mayoritario que corresponde a un flavonol de tipo glicósido de Kaempferol y se identificó el flavanol catequina a bajas concentraciones. Por medio de Cromatografía de Gases-Espectrometría de Masas (CG-MS) se identificaron los sesquiterpenos $\beta$-elemeno, E-cariofileno, aromadendreno, humuleno, gama-cadineno, D-germacreno, biciclogermacreno y los poli terpenos Di- $\alpha$-Tocoferol y $\beta$-sitosterol. Nuestros resultados sugieren que la evaluación de los fenoles puede desempeñar un papel importante como parámetro de selección en programas de mejora y conservación, si se complementan con las prácticas convencionales de mejoramiento genético.

Palabras clave: Cedrela odorata, hojas, fenoles, limonoides, HPLC, GC-MS.

\section{REFERENCIAS}

Andersen, O. M., \& Markham, K. R. (Eds.) (2006). Flavonoids chemistry, biochemistry and applications. Boca Raton: CRC Press.

Asekun, O. T., \& Ekundayo, O. (1999). Constituents of the leaf essential oil of Cedrela odorata L. from Nigeria. Flavour and Fragrance Journal, 14, 390-392.

Avalos, G. A., \& Pérez-Urria, C. E. (2009). Metabolismo secundario de plantas. Reduca (Biología), 2(3), 119-145.
Barboza, J., Hilje, L., Durón, J., Cartín, V., \& Calvo, M. A. (2010). Actividad fagodisuasiva y sistémica de una formulación derivada de un extracto de ruda (Ruta chalepensis, Rutaceae) sobre larvas de Hypsipyla grandella (Lepidoptera: Pyralidae). Revista de Biología Tropical, 58,15-29.

Campos, M. G., \& Markham, K. R. (2007). Structure information from HPLC and on-line measured absorption spectra-flavone, flavonols andphenolic acids. Portugal: Coimbra University Press.

Céspedes, L. C., Calderón, S. J., Lina, L., \& Aranda, E. (2000). Growth inhibitory effects on fall armyworm Spodopetera frugiperda of some limonoids isolated from Cedrela spp. (Meliaceae). Journal of Agricultural and Food Chemistry, 48, 1903-1908.

Champagne, D. E., Koul, O., Isman, M. B., Scudder, G. G., \& Towers, G. H. (1992). Biological activity of limonoids from the Rutales. Phytochemistry, 31, 377-394.

Cornelius, J. P., \& Watt, A. D. (2003). Genetic variation in a Hypsipyla-attacked clonal trial of Cedrela odorata under two pruning regimes. Forest Ecology and Management, 183, 341-349.

Da Silva, M. F. G. F., Agostinho, S. M., de Paula, J. R., Neto, J. O., Castro-Gamboa, I., Rodrigues, F. E., Fernandes, J. B., \& Vieira, P. C. (1999). Chemistry of Toona ciliata and Cedrela odorata graft (Meliaceae): chemosystematic and ecological significance. Pure Applied Chemistry, 71(6), 1083-1087.

Dai, J., Yaylayan, V. A., Raghavan, G. V., \& Parè, J. R. (1999). Extraction and colorimetric determination of azadirachtin-related limonoids in neem seed kernel. Journal of Agricultural and Food Chemistry, 47(9), 3738-3742.

De la Torre, A., López, C., Yglesias, E., \& Cornelius, J. P. (2008). Genetic (AFLP) diversity of nine Cedrela odorata populations in Madre de Dios, southern Peruvian Amazon. Forest Ecology and Management, 255, 334-339.

De Paula, J., Vieira, I. J., Da Silva, M. F. G. F., Fo, E. R., Fernandes, J. B., Vieira, P. C., Pinheiro, A. L., \& Vilela, E. F. (1997). Sesquiterpenes, triterpenoids, limonoids and flavonoids of Cedrela odorata graft and speculations on the induced resistance against Hypsipyla grandella. Phytochemistry, 44, 1449-1454.

González-Coloma, A., Reina, M., Sáenz, C., Lacret, R., Ruiz-Mesia, L., Arán, V. J., Sanz, J., \& Martínez-Díaz, R. A. (2012). Antileishmanial, antitrypanosomal, and cytotoxic screening of ethnopharmacologically selected Peruvian plants. Parasitology Research, 110, 1381-1392.

Hammer, Ø., Harper, D.A.T., \& Ryan, P. D. (2001). PAST: Paleontological statistics software package for education and data analysis. Palaeontologia Electronica 4(1). Recuperado de http://palaeo-electronica. org/2001_1/past/issue1_01.htm 
Harborne, J. B. (1973). Phytochemical methods, London: Chapman and Hall.

Harborne, J. B. (2001). Twenty-five years of chemical ecology. Natural Product Reports, 18, 361-379.

Heimler, D., Vignolini, P., Dini, M., \& Romani, A. (2005). Rapid test to assess the antioxidant activity of Phaseolus vulgaris L. dry beans. Journal of Agricultural and Food Chemistry, 53, 3053-3056.

Hilje, L., \& Cornelius, J. (2001). ¿Es inmanejable Hypsipyla grandella como plaga forestal? Manejo Integrado de Plagas, 61, 1-4.

Kipassa, N. T., Iwagawa, T., Okamura, H., Doe, M., Morimoto, Y., \& Nakatani, M. (2008). Limonoids from the stem bark of Cedrela odorata. Phytochemistry, 69, 1782-1787.

Larrea, R. G., De los Santos Posadas, H. M., \& Hernández, J. I. V. (2008). Crecimiento y rendimiento maderable de Cedrela odorata L. y Tabebuia donnell-smithii Rose en San José Chacalapa, Pochutla, Oaxaca. Madera y Bosques, 14(2), 65-82.

Lee, I. S., Wei, C., Thuong, P. T., Song, K. S., Seong, Y. H., \& Bae, K. H. (2006). Antioxidant constituents from the leaves of Cedrela sinensis A. Juss. Korean Journal of Medicinal Crop Science, 14(5), 267-272.

Macías-Sámano, J. E. (2001). Interacciones químicas entre Hypsipyla grandella y sus plantas hospedantes. Manejo Integrado de Plagas, 60, 15-21.

Maia, B. H., Paula, J. R. D., Sant'Ana, J., Silva, M. F. G. F., Fernandes, J. B., Vieira, P. C., Costa, M. S. S., Oashi, O. S., \& Silva, J. N. M. (2000). Essential oils of Toona and Cedrela species (Meliaceae): taxonomic and ecological implications. Journal of the Brazilian Chemical Society, 11(6), 629-639.

Martins, A. P., Salgueiro, L. R., da Cunha, A. P., Vila, R., Cañigueral, S., Tomi, F., \& Casanova, J. (2003). Chemical composition of the bark oil of Cedrela odorata from S. Tome and Principe. Journal of Essential Oil Research, 15, 422-424.

Millán-Orozco, L., Corredoira, E., \& San José, M. D. C. (2011). In vitro rhizogenesis: histoanatomy of Cedrela odorata (Meliaceae) microcuttings. Revista de Biología Tropical, 59, 447-453.

Muellner, A. N., Pennington, T. D., \& Chase, M. W. (2009). Molecular phylogenetics of Neotropical Cedreleae (mahogany family, Meliaceae) based on nuclear and plastid DNA sequences reveal multiple origins of "Cedrela odorata". Molecular Phylogenetics and Evolution, 52, 461-469.

Navarro, C., Ward, S., \& Hernández, M. (2002). The tree Cedrela odorata (Meliaceae): a morphologically subdivided species in Costa Rica. Revista de Biologia Tropical, 50, 21-30.

Newton, A. C., Cornelius, J. P., Mesen, J. F., \& Leakey, R. R. B. (1995). Genetic variation in apical dominance of Cedrela odorata seedlings in response to decapitation. Silvae Genetica, 44(2), 146-149.

Newton, A. C., Leakey, R. R. B., \& Mesean, J. F. (1993). Genetic variation in mahoganies: Its importance, utilization and conservation. Biodiversity and Conservation, 2(2), 114-126.

Newton, A. C., Watt, A. D., Lopez, F., Cornelius, J. P., Mesén, J. F., \& Corea, E. A. (1999). Genetic variation in host susceptibility to attack by the mahogany shoot borer, Hypsipyla grandella (Zeller). Agricultural and Forest Entomology, 1(1), 11-18.

Omar, S., Zhang, J., MacKinnon, S. A., Leaman, D., Durst, T., Philogene, B. J. R., Arnason, J. T., SánchezVindas, P. E., Poveda, L., Tamez, P. A., \& Pezzuto, J. M. (2003). Traditionally-used antimalarials from the Meliaceae. Current Topics in Medicinal Chemistry, 3, 133-139.

Patiño, V. F. (1997). Genetic resources of Swietenia macrophylla and Cedrela odorata in the neotropics: Priorities for coordinates actions. Forest Genetic Resources, 25, 20-32.

Pereyra, E. M. J., Inga, G. G. J., Santos, M. M., \& Arisméndiz, R. (2014). Potencialidad de Cedrela odorata (Meliaceae) para estudios dendrocronológicos en la selva central del Perú. Revista de Biología Tropical, 62, 783-793.

Pérez-Flores, J., Eigenbrode, S. D., \& Hilje-Quiroz, L. H. (2012). Alkaloids, Limonoids and Phenols from Meliaceae Species Decrease Survival and Performance of Hypsipyla grandella Larvae. American Journal of Plant Sciences, 3, 988-994.

Pérez-Salicrup, D. R., \& Esquivel, R. (2008). Tree infection by Hypsipyla grandella in Swietenia macrophylla and Cedrela odorata (Meliaceae) in Mexico's southern Yucatan Peninsula. Forest Ecology and Management, 255, 324-327.

Ramputh, A., Teshome, A., Bergvinson, D. J., Nozzolillo, C., \& Arnason, J. T. (1999). Soluble phenolic content as an indicator of sorghum grain resistance to Sitophilus oryzae (Coleoptera: Curculionidae). Journal of Stored Products Research, 35(1), 57-64.

Rosales-Castro, M., González-Laredo, R. F., Rocha-Guzmán, N. E., Gallegos-Infante, J. A., Peralta-Cruz, J., \& Karchesy, J. J. (2009). Evaluación química y capacidad antioxidante de extractos polifenólicos de cortezas de Pinus cooperi, P. engelmannii, P. leiophylla y P. teocote. Madera y Bosques, 15(3), 87-105.

Rosales-Castro, M., González-Laredo, R. F., Rocha-Guzmán, N. E., Gallegos-Infante, J. A., Rivas-Arreola, M. J., \& Karchesy, J. J. (2012). Antioxidant activity of fractions from Quercus sideroxyla bark and identification of proanthocyanidins by HPLC-DAD and HPLC-MS. Holzforschung, 66(5), 577-584. 
Sánchez, M. V., Salazar, G. J., Vargas, H. J. J., López, U. J., \& Jasso, M. J. (2003). Parámetros genéticos y respuesta a la selección en características del crecimiento de Cedrela odorata L. Revista Fitotecnia Mexicana, 26(1), 19-27.

Secretaría del Medio Ambiente y Recursos Naturales (SEMARNAT). (2010). Norma Oficial Mexicana NOM-059-SEMARNAT-2010, Protección ambientalEspecies nativas de México de flora y fauna silvestres: Categorías de riesgo y especificaciones para su inclusión, exclusión o cambio-Lista de especies en riesgo. Diario Oficial de la federación. Instituto Nacional de ecología. México.

Sidhu, O. P., Kumar, V., \& Behl, H. M. (2004). Variability in triterpenoids (nimbin and salanin) composition of neem among different provenances of India. Industrial Crops and Products, 19(1), 69-75.

Silva, E. M., Souza, J. N. S., Rogez, H., Rees, J. F., \& Larondelle, Y. (2007). Antioxidant activities and polyphenolic contents of fifteen selected plant species from the Amazonian region. Food Chemistry, 101, 1012-1018.

Soto, F., Hilje, L., Mora, G. A., \& Carballo, M. (2011). Phagodeterrence by Quassia amara (Simaroubaceae) wood extract fractions on Hypsipyla grandella (Lepidoptera: Pyralidae) larvae. Revista de Biología Tropical, 59, 487-499.

StatSoft, Inc. (2004). STATISTICA (data analysis software system), version 7. www.statsoft.com

Taylor, D. A. H. (1981). Chemotaxonomy: the occurrence of limonoids in the Meliaceae. In T. D. Pennington (Eds.), Flora Neotropica Monograph Number 28 (pp. 450-459). New York: New York Botanical Garden.

Villanueva, H. E., Tuten, J. A., Haber, W. A., \& Setzer, W. N. (2009). Chemical composition and antimicrobial activity of the bark essential oil of Cedrela odorata from Monteverde, Costa Rica. Der Pharma Chemica, 1(2), 14-18.

Waterman, P. G., \& Mole, S. (1994). Analysis of Phenolic Plant Metabolites. Methods in Ecology. Boston: Blackwell Scientific Publications. 